\title{
Einarbeitung mit Struktur
}

\author{
Christian Stimming, Patrick Dormann, Philippe Valentin, Stefan Reimers
}

\author{
Wie kann die Einarbeitung unerfahrener Mitarbeiter in Notaufnahmeabteilungen - \\ vor allem für Berufsanfänger ohne Praxiserfahrung - strukturiert und organisiert werden? \\ Ein Erfahrungsbericht aus Sicht der Praxisanleitung.
}

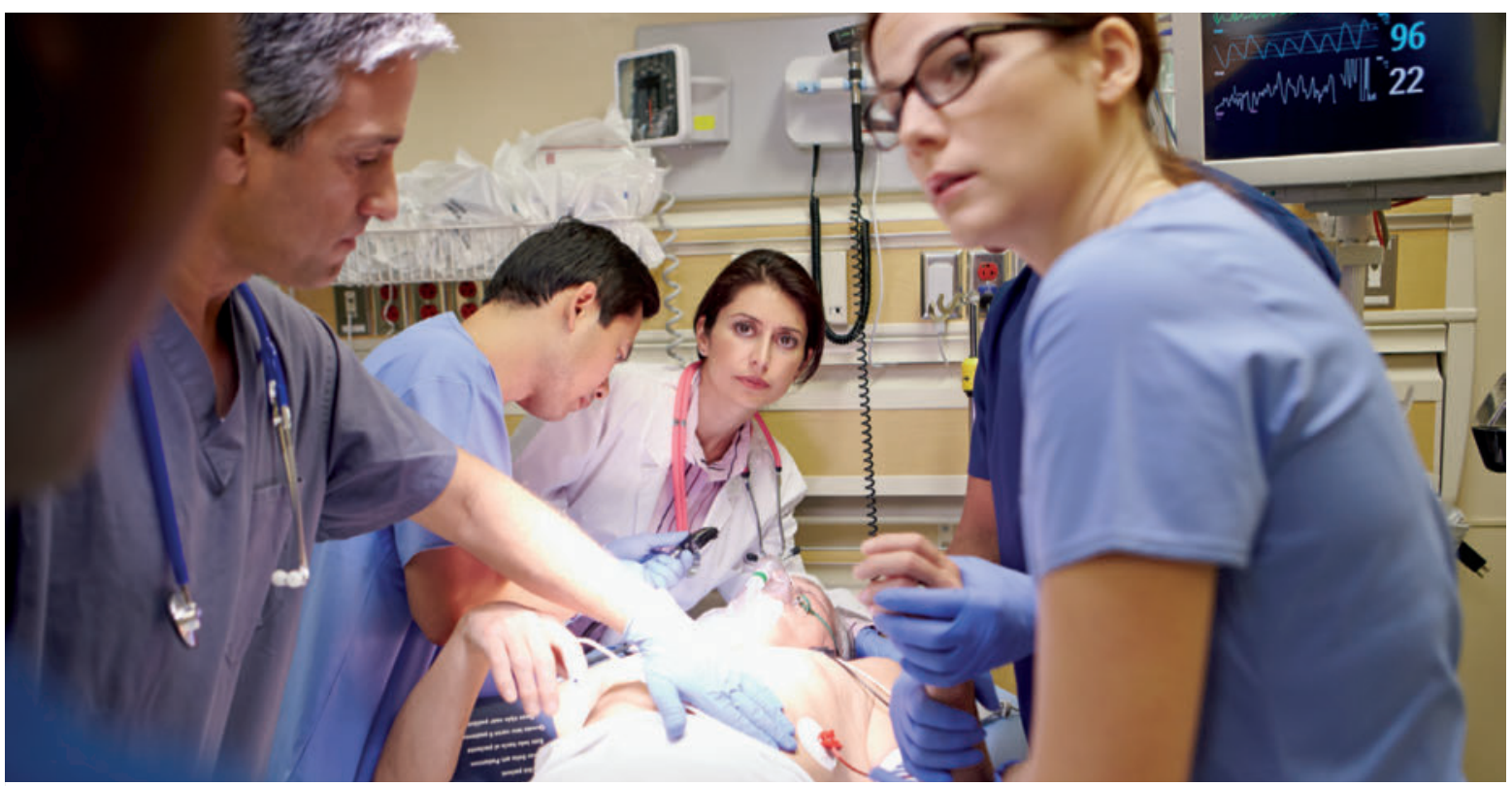

Eine strukturierte Einarbeitung neuer Mitarbeiter ist Voraussetzung für ein reibungsloses Funktionieren aller Abläufe in der Notaufnahme. (Quelle: Monkey Business_AdobeStock; Symbolbild)

Das Handlungsspektrum von Notaufnahmeabteilungen erstreckt sich von der Behandlung schwerverletzter und schwerstkranker Patienten bis hin zur Behandlung von Patienten, die von ihrem Hausarzt oder dem hausärztlichen Notdienst behandelt werden könnten. Dabei erhoffen sich der Notfallpatient sowie dessen An- und Zugehörige in erster Linie die Linderung der akuten Beschwerden, eine zügige und zielsichere Diagnose sowie eine rasche und kompetente Therapie [1]. Ziel muss es sein, trotz der in den letzten Jahren kontinuierlich steigenden Patientenzahlen in Notaufnahmeabteilungen keinen schwerstkranken Patienten zu übersehenn. Daher sollten alle Patienten in Zentralen Notaufnahmen (ZNA) ersteingeschätzt werden. Diese Ersteinschätzung sollte von geschulten Mitarbeitern des Pflegedienstes durchgeführt werden [2]. Doch erstreckt sich das Handlungsfeld von Pflegenden in der Notaufnahme auf einen weitaus größeren Tätigkeitsbereich, dazu gehören die Linderung/Unterstützung bei der Heilung der subjektiven Beschwerden von Patienten und das Erkennen ihrer Bedürfnisse. Hinzu kommen die pflegerische Versorgung und die Betreuung von Angehörigen, das Einleiten/Interpretieren diagnostischer Maßnahmen und der Beginn der indizierten Therapie zur Sicherstellung der Behandlungsqualität [3]. Dies ist insbesondere für neue Kollegen ohne Berufserfahrung eine anspruchsvolle Aufgabe, da sie hierfür in ihrer Ausbildung nicht vorbereitet werden [4]. In der Ausbildungsrichtlinie für „Gesundheits- und (Kinder-)Krankenpflege“ in Nordrhein-Westfalen sind beispielsweise nur einzelne Unterrichtsstunden hinterlegt, die Pflegende auf die Tätigkeit in einer Zentralen Notaufnahme vorbereiten [5].

Daher ist es eine unumgängliche Aufgabe der Führungskräfte und Praxisanleiter von Notaufnahmen, neue Kollegen strukturiert und umfassend auf ihr neues Aufgabengebiet vorzubereiten. Die Einarbeitung ist auf ihre Wirksamkeit hin zu evaluieren, und auch nach der geplanten Einarbeitung sind weitere Fortbildungsangebote zu ermöglichen, um eine kontinuierliche Steigerung der pflegerischen Kompetenzen bzw. des Wissensstands zu gewährleisten. Mit dem Ziel einer kontinuierlichen und hohen Pflegequalität trotz teilweise geringer Berufserfahrung 
bei neuen Kollegen wurde in der ZNA der Uniklinik Köln ein neues Einarbeitungskonzept entwickelt.

\section{Die Notaufnahme der Uniklinik Köln}

Die Zentrale Notaufnahme der Uniklinik Köln (UKK) versorgt jährlich ca. 45.000 Menschen und hat rund 60.500 Patientenkontakte im Jahr. Als Haus der Maximalversorgung ist die UKK Bestandteil des Traumanetzwerks Region Köln als überregionales Traumazentrum und Bestandteil des Kölner Infarktmodells (KIM) mit einer angebundenen zertifizierten Chest Pain Unit. In der Notaufnahme ( $\triangleright$ Info) werden alle instabilen oder polytraumatisierten Patienten interprofessionell betreut. Die Notaufnahme wird ärztlicherseits durch die Klinik für Innere Medizin II (Nephrologie, Rheumatologie, Diabetologie und Allgemeine Innere Medizin Uniklinik Köln) geführt. Die Ersteinschätzung von Patienten geschieht mittels Manchester-Triage-System.

Durch die Zuweisung der Notaufnahme innerhalb des Pflegedienstes zum Bereich der Intensivpflege ist es den Mitarbeitern der ZNA möglich, an der hausinternen Fachweiterbildung Intensivpflege und Anästhesie teilzunehmen. Daher haben etwa 30 Prozent der Pflegekräfte diese fachliche Zusatzqualifikation. Aktuell werden die Mitarbeiter zusätzlich durch die Weiterbildung Notfallpflege qualifiziert.

Mit den teilzeitbeschäftigten Kollegen zählt das pflegerische Team derzeit 42 Mitarbeiter. Darunter sind zehn Pflegekräfte mit Fachweiterbildung Intensivpflege und Anästhesie, zwei mit der Fachweiterbildung Notfallpflege nach DKG, sieben Praxisanleiter und ein Absolvent des Studiengangs ANP, zwei Mitarbeiter befinden sich in einem pflegebezogenen Bachelorstudiengang.

\section{Einarbeitung als zielorientierter Prozess}

In der Einarbeitung im Kontext Notaufnahme geht es daher vor allem darum, dem neuen Beschäftigten Sicherheit im neuen Arbeitsumfeld zu vermitteln, um so die Qualität im Sinne der Patientensicherheit zu gewährleisten. Die Einarbeitung hat gezielt zu erfolgen und muss didaktisch vorbereitet und geplant werden, daher kann sie an den PDCA-Zyklus angelehnt werden:

- Plan= Wer übernimmt die Einarbeitung? Welche Vorerfahrungen hat der neue Mitarbeiter? Interne Formalien (z. B. Schlüsselantrag, Freischaltungen beantragen etc.)

- Do=Führe die Einarbeitung durch

- Check= Evaluiere die Einarbeitung und die Fortschritte gemeinsam mit dem Einzuarbeitenden, Praxisanleitung und Teamleitung

- $\mathbf{A c t}=$ Führe Anpassungen durch, abgeleitet aus der jeweiligen Evaluation (z. B. Veränderung der Lehrmethoden oder Verlängerung der Einarbeitungszeit)
RÄUMLICHE, PERSONELLE UND STRUKTURELLE

GEGEBENHEITEN DER ZNA DER UNIKLINIK KÖLN

- Drei Schockräume

- Neun Behandlungsräume

- Zwei Funktionsräume

- Unmittelbare Nähe zur Notfallradiologie und Hubschrauberlandeplatz

- Einbindung im Traumanetzwerk Region Köln und Kölner Infarktmodell

- Pflegende sind fächerübergreifend weitergebildet (z. B. Fachweiterbildungen in Notfallpflege, Anästhesie und Intensivpflege, Praxisanleiter für Pflegeberufe, Wundmanagement, pädiatrische Intensivpflege)

- Pflegende sind multiprofessionell tätig

- Angegliederte Notaufnahmestation mit zehn Betten zur interdisziplinären Nutzung

\section{Problemdarstellung}

Zwischen Oktober 2016 und Dezember 2017 wurden insgesamt zwölf neue Mitarbeiter eingearbeitet, darunter allein fünf im Oktober 2016. Die neuen Kollegen hatten mehrjährige Berufserfahrung in der Allgemeinpflege, der Anästhesie oder Psychiatrie, zum anderen gab es auch Kollegen mit nur wenig Berufserfahrung. Innerhalb der vorgesehenen sechs Wochen Einarbeitungszeit mussten den neuen Mitarbeitern daher nicht nur die strukturellen Konzepte der Uniklinik Köln vermittelt werden, sondern auch fachliche Inhalte unter Berücksichtigung des speziellen Arbeitsplatzes Notaufnahme.

\section{Rückblick zur bisherigen Einarbeitung - was sollte sich ändern?}

Die Einarbeitung neuer Mitarbeiter wurde durch die pflegerische Teamleitung der Zentralen Notaufnahme an die Praxisanleiter delegiert. Jedoch gab es bisher kein spezielles Einarbeitungskonzept seitens der Pflegedirektion für den Funktionsbereich Notaufnahme. Die bisherigen Einarbeitungen waren daher weder systematisch noch durch ein pädagogisches Konzept hinterlegt und daher stark abhängig vom jeweiligen Praxisanleiter. Trotz dieser Problematik konnte man aufgrund der hohen fachlichen Expertise der Praxisanleiter von einer inhaltlich versierten Einarbeitung ausgehen.

Die bisherige Einarbeitung erfolgte allerdings immer im direkten Patientenkontakt. Das führte zu einer Doppelbelastung für den Praxisanleiter und den neuen Mitarbeiter, da zum einen weiterhin eine adäquate Patientenversorgung gewährleistet werden musste, zum anderen aber parallel gelehrt und gelernt werden sollte.

\section{Das neue Einarbeitungskonzept}

Um die Diskrepanz einer nicht-strukturierten Einarbeitung zu beheben, haben die Praxisanleiter der Uniklinik Köln ein 

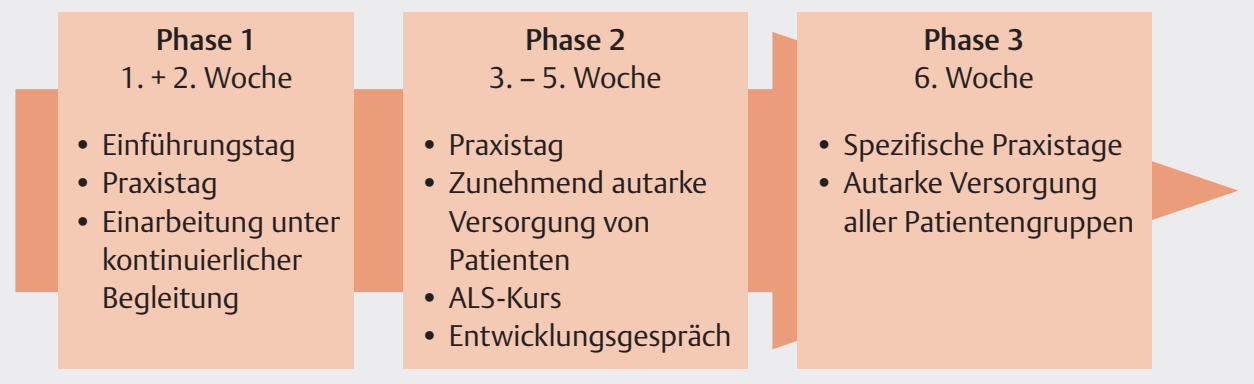

- Abb. 1 Die drei Phasen der Einarbeitung.

> Tab. 1 Mögliche Inhalte der Einführungs- und Praxistage.

\begin{tabular}{|c|c|c|}
\hline Einführungstag & Erster Praxistag & Zweiter Praxistag \\
\hline $\begin{array}{l}\text { - Geräteeinweisungen } \\
\text { - Räumlichkeiten } \\
\text { - Fluchtwege und Feuer- } \\
\text { löscheinrichtungen } \\
\text { - Fachabteilungen } \\
\text { - Versorgungsprozess } \\
\text { - Erstkontakt/Erstein- } \\
\text { schätzung }\end{array}$ & $\begin{array}{l}\text { - Stroke in der ZNA } \\
\text { - Polytraumaver- } \\
\text { sorgung } \\
\text { - Chest Pain Unit } \\
\text { - Human Factors }\end{array}$ & $\begin{array}{l}\text { - Spezielle Krankheits- } \\
\text { bilder } \\
\text { - Themen der Einzu- } \\
\text { arbeitenden } \\
\text { - Verhalten im ManV } \\
\text { - Erweiterte Geräte- } \\
\text { einweisungen (z. B. } \\
\text { Beatmungsgerät) }\end{array}$ \\
\hline
\end{tabular}

\section{PFLEGE FÜR PFLEGE}

- Internes Fortbildungsprogramm mit Kurzpräsentationen

- Alle zwei Wochen

- Durchführung vor dem Spätdienst und nach dem Frühdienst mit je 20 Minuten

- Referenten sind grundsätzlich pflegerische Kollegen (daher auch der Name „Pflege für Pflege“)

\section{Themenbeispiele:}

- EKG-Veränderungen

- ABC-Schema

- Spezielles Monitoring bei Kindern

- BGA-Analyse

- Gipsschienen

\section{Phase 1}

Die erste Phase ist gekennzeichnet durch eine kontinuierliche Einarbeitung mit einer bzw. zwei festen Bezugspersonen. Eröffnet wird diese Phase mit einem Einführungstag und einem Praxistag. Diese finden außerhalb der Patientenversorgung statt und können dadurch optimal genutzt werden, um theoretisches Grundlagenwissen zu vermitteln. Die Themen ( $\triangleright$ Tab. 1) des Einführungs- und Praxistages werden dann in der weiteren Einarbeitung praktisch angewandt, um einen Theorie-Praxis-Transfer zu gewährleisten.

\section{Phase 2}

Die zweite Phase beinhaltet einen Praxistag außerhalb der Patientenversorgung. Hier werden die Themen individuell auf den Einzuarbeitenden abgestimmt. Es können weitere theoretische Grundlagen besprochen, aber auch praktische Ansätze trainiert werden. Eine Vertiefungsmöglichkeit stellen z. B. seltene Krankheitsbilder oder Symptomkomplexe dar.

Nach diesem Praxistag werden die neuen Kollegen zwar weiterhin kontinuierlich begleitet, übernehmen die Versorgung von Patienten aber im Rahmen ihrer Kompetenzen zunehmend autark und erhalten ein Feedback dazu. Darüber hinaus ist die Teilnahme an einem Advanced-Life-Support-Kurs im „Kölner interprofessionellen Skillslab und Simulationszentrum“ (KISS) geplant. Sollte es in dieser Phase absehbar werden, dass die begleitete Zeit verlängert werden muss, wird dies zwischen Praxisanleitung, Teamleitung und neuem Mitarbeiter im Rahmen eines Entwicklungsgesprächs besprochen.

neues Einarbeitungskonzept entworfen. Die Voraussetzung, neue Mitarbeiter einzuarbeiten, ist seither eine Weiterbildung im Bereich der Anästhesie und Intensivpflege und/ oder im Bereich der Praxisanleitung sowie mehrjährige Erfahrung im Notaufnahmesetting. Die Mehrzahl der einarbeitenden Pflegenden verfügt über beide Weiterbildungen, womit eine hohe fachliche und pädagogische Kompetenz der Anleiter sichergestellt wurde. Es wurden Einführungsund Praxistage erarbeitet, um theoretisches Hintergrundwissen zu schaffen. Daraus entstand ein drei Phasen-Konzept ( $\triangleright$ Abb. 1) mit einer Dauer von sechs Wochen:

\section{Phase 3}

In der dritten Phase der Einarbeitung werden spezifische Praxistage je nach Wissensstand und Vorerfahrungen des Mitarbeiters angeboten. Des Weiteren versorgt der neue Mitarbeiter Patienten weitestgehend autark, wobei die Pflegehandlungen weiterhin durch den Praxisanleiter beobachtet und gemeinsam mit dem Einzuarbeitenden evaluiert werden. Auch nach Abschluss der Einarbeitungszeit steht der Praxisanleiter dem neuen Kollegen weiterhin als Ansprechpartner zur Verfügung. 
Eine individuelle Anpassung der Inhalte in den einzelnen Phasen der Einarbeitung an die Vorerfahrung der neuen Kollegen ist für die Akzeptanz des Einarbeitungskonzepts von hoher Bedeutung. Die Inhalte der Einarbeitung müssen daher an die jeweiligen Vorerfahrungen (Erfahrung in der Intensivpflege, Erfahrung in der Notaufnahmepflege, Erfahrung aus anderen pflegerischen Bereichen) der neuen Mitarbeiter adaptiert werden.

\section{Zusammenfassung}

Die fachliche Einarbeitung ist ein wichtiger Baustein zur Mitarbeiterentwicklung und -bindung. Aus der gemeinsamen Evaluation zwischen Teamleitung, Praxisanleitung und neu eingearbeiteten Kollegen ging hervor, dass die Einarbeitung durch das neue Konzept zwar stringenter und auf die individuellen Vorerfahrungen der einzelnen Mitarbeiter angepasster ist, jedoch im Sinne des lebenslangen Lernens sowie der Mitarbeiterbindung und -entwicklung durch weitere Fortbildungsmaßnahmen nach Beendigung der Einarbeitungszeit unterstürzt werden muss.

Aus dieser Idee entstanden das Fortbildungskonzept - Pflege für Pflege sowie die Einführung spezieller Praxistage in an die Notaufnahme angrenzenden Versorgungsbereichen ( $\triangleright$ Ein Tag auf ITS).

\section{INTERESSANTE LINKS}

- www.nephrologie.uk-koeln.de/klinik/zentralenotaufnahme

- www.kiss-uk-koeln.de

\section{Autorinnen/Autoren}

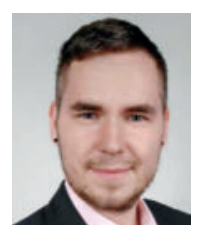

\section{Christian Stimming}

Gesundheits- und Krankenpfleger, Praxisanleiter für Pflegeberufe, Qualitätsbeauftragter und interner Auditor (DGQ). Mitglied in DGINA; DBfK, BLGS und DGF. Student der Pflegewissenschaften (B.Sc.) an der Katholischen Hochschule NRW/Köln, Uniklinik Köln Zentrale Notaufnahme.

E-Mail: Christian.Stimming@uk-koeln.de

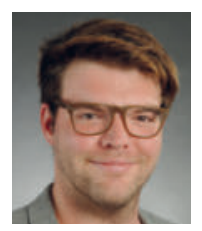

\section{Patrick Dormann}

Fachkrankenpfleger für Intensivpflege und Anästhesie, Krankenpfleger für die Notfallpflege (DKG), B.A. ANP, Praxisanleiter. Sprecher des Aktionsbündnisses Notfallpflege, Mitglied DGINA, DGF, DIVI. Caritas-Akademie Köln-Hohenlind und Zentrale Notaufnahme der Uniklinik Köln.

\section{EIN TAG AUF ITS}

... als Beispiel eines Praxistages im angrenzenden Versorgungsbereich

- Gemeinsamer Arbeitstag auf Intensivstation mit Praxisanleitung

- Theoretische Grundlagen (vier Stunden)

- Praktische Durchführung (vier Stunden)

\section{Mögliche Themen:}

- Absaugen

- Extubation (Intubation)

- Umgang mit Zu- und Ableitungen

- Nasen- und Mundpflege bei intubierten Patienten

- Tubusumlagerung

- Umgang mit Magensonde

- Medikamentenkunde

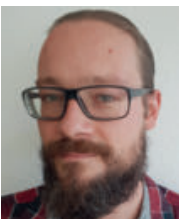

\section{Philippe Valentin}

Fachkrankenpfleger für Intensivpflege und Anästhesie, Krankenpfleger für die Notfallpflege (DKG). Teamleitung Uniklinik Köln Zentrale Notaufnahme.

\section{Stefan Reimers}

Pflegedienstleitung Intensivpflege, Fachkrankenpfleger für Intensivpflege und Anästhesie, Dipl. Pflegewirt (FH), M.A. Pflegemanagement, Uniklinik Köln.

Literatur

[1] Moecke H, Lackner CK, Klöss T. Das ZNA-Buch - Konzepte, Methoden und Praxis der Zentralen Notaufnahme. Berlin: Medizinisch Wissenschaftliche Verlagsgesellschaft; 2011

[2] Windle J, Mackway-Jones K, Marsden J. Ersteinschätzung in der Notaufnahme - Das Manchester-Triage-System. 2. Aufl. Bern: Hans Huber; 2010

[3] Dormann P, Wedler K, Machner M et al. Notfallpflege - was ist das eigentlich? intensiv 2017, 25(06): 293-8

[4] DGiNA AG Pflege (Wedler K, Friesdorf M, Dietz-Wittstock M). Empfehlungen zur Fachweiterbildung Notfallpflege. 2014. Online unter bit.ly/2DPvfdv, letzter Zugriff 21.11.2018

[5] Oelke U. Richtlinie für die Ausbildung in der Gesundheits- und Krankenpflege sowie in der Gesundheits- und Kinderkrankenpflege. Im Auftrag des Ministeriums für Gesundheit, Soziales, Frauen und Familie des Landes Nordrhein-Westfalen. 1998. Anpassung: Gertrud Hundenborn/Cornelia Kühn, Deutsches Institut für angewandte Pflegeforschung e. V., November 2003. Online unter bit.ly/2OVbbHz, letzter Zugriff 21.11.2018

[6] Lobinger W, Haas J, Groß HA. Qualitätsmanagement in der Pflege. 2. Aufl. München: Hanser; 2013

Bibliografie

DOI https://doi.org/10.1055/a-0753-4518

intensiv 2019; 27: 12-15

(c) Georg Thieme Verlag KG Stuttgart · New York ISSN 0942-6035 